\title{
INVESTIGACIONES
}

\section{Juegos cooperativos con jóvenes en situación de vulnerabilidad social: la sistematización de una experiencia}

\author{
Cooperative games with young people in situations of social vulnerability: \\ the systematization of an experience \\ Jogos cooperativos com jovens em situação de vulnerabilidade social: \\ a sistematização de uma experiencia \\ Natalie García ${ }^{a}$,Abinaira Piassa ${ }^{a}$, Marcio Ribeiro $^{a}$ \\ ${ }^{a}$ Centro Universitario Salesiano de São Paulo (UNISAL)/Americana/Brasil. \\ nathygarcia29@gmail.com,nayrapiassa@gmail.com, ribmarcio@yahoo.com.br
}

\begin{abstract}
RESUMEN
Para trabajar los Juegos Cooperativos como posibilidad de Educación Popular, este Proyecto de Acción Educativa, disciplina que permea toda la formación del curso de Pedagogía en el Centro Universitario Salesiano de São Paulo - UNISAL / Americana / Brasil, buscó desarrollar actividades desde una perspectiva lúdica capaz de contribuir a que los niños ejerciten la reflexión sobre las actitudes cooperativas y su práctica. Las actividades aquí sistematizadas se realizaron con niños de 6 a 13 años, que asisten al proyecto "Escuela de la Familia", inserto en una escuela pública del municipio de Americana. El principal objetivo de este trabajo fue contribuir con una formación educativa que traiga el equilibrio entre competitividad y cooperación, constituyendo así una práctica que va más allá de la educación escolar. La metodología utilizada se basó en los escritos de Oscar Jara (2006), quien en su tesis defiende cinco tiempos de sistematización para la conclusión de un buen trabajo de campo. A saber: 1) el punto de partida, que requiere la participación activa de los autores, además de registros de experiencias; 2) las preguntas iniciales que en el presente trabajo fueron: ¿Cuál es la comprensión de los niños sobre los juegos cooperativos? ¿Permite esta práctica nuevos horizontes? ¿Cuáles? ¿Es posible tener un equilibrio entre la cooperación y la competencia para los niños? ¿Cómo puede la práctica de la cooperación ayudar a los niños en el desarrollo de su vida social? 3) la recuperación del tiempo vivido, que busca describir todo el proceso por el que atraviesan las actividades y experiencias.; 4) las reflexiones de fondo, en las que se busca profundizar en la comprensión y discusión de los autores y participantes sobre las acciones vividas, que fueron desarrollados en todos los procesos. Una de las percepciones que trajo la realización de las actividades es que la cooperación está en nosotros, solo tenemos que trabajar en ello para que sea evidente. 5) los puntos de llegada, que son el análisis de todo el aprendizaje en el proceso. Los resultados finales de esta intervención educativa demostraron que los niños mostraron pequeñas transformaciones, sin embargo, significativas en cuanto a sus comportamientos, y sus prácticas con los demás compañeros participantes en los juegos y también con las personas en general. Respondiendo a las inquietudes iniciales de este proyecto, consideramos que la realización de estas actividades trajo cercanía entre los participantes y acciones colaborativas, llevándonos a la convicción de que el cambio en el comportamiento de los niños traspasó los límites de los muros escolares.
\end{abstract}

Palabras claves: Juegos Cooperativos, Sistematización de la experiencia, Educación Popular.

ABSTRACT
In order to work on the Cooperative Games as a possibility of Popular Education, this Educational Action Project,
a discipline that permeates the entire formation of the Pedagogy course at the Salesian University Center of São
Paulo - UNISAL / Americana / Brazil, sought to develop activities within from a playful perspective capable of 
contributing for children to exercise reflection on cooperative attitudes and their practice. The activities systematized here were carried out with children from 6 to 13 years old, who attend the "Family School" project, inserted in a state school in the municipality of Americana/SP/Brazil. The main objective of this work was to contribute with an educational formation that brought the balance between competitiveness and cooperation, thus constituting a practice that goes beyond school education. The methodology used was based on the writings of Oscar Jara (2006), who in his thesis defends five times of systematization for the conclusion of a good fieldwork. They are: 1) the starting point, which requires active participation by the authors, in addition to records of experiences; 2) the initial questions that in the present work were: What is the children's understanding of cooperative games? Does this practice enable new horizons? Which ones are they? Is it possible to have a balance between cooperation and competition for children? How can the practice of cooperation help children in the development of their social life? 3) the recovery of the time lived, which seeks to describe the entire process through which the activities and experiences go through 4) the background reflections, in which it seeks to deepen the understanding and discussion of the authors and participants about the actions experienced, which were all the processes developed. One of the perceptions that the realization of this game brought is that cooperation is in us, we just need to work on it to make it evident; 5) the arrival points, which are the analysis of all the learning in the process. The final results of this educational intervention demonstrated that the children showed small, however, significant transformations with regard to their behaviors, and their practices with the other colleagues participating in the games and also with people in general. Responding to the initial concerns of this project, we consider that the realization of these activities brought proximity between the participants and collaborative actions, leading us to the conviction that the change in the children's behavior went beyond the limits of the school walls.

Key words: Cooperative Games, Systematization of experience, Popular Education.

\section{RESUMO}

Com o objetivo de trabalhar os Jogos Cooperativos como possibilidade de Educação Popular, este Projeto de Ação Educativa, disciplina que permeia toda a formação do curso de Pedagogia do Centro Universitário Salesiano de São Paulo - UNISAL / Americana / Brasil, buscou desenvolver atividades dentro de uma perspectiva lúdica capaz de contribuir para que as crianças exerçam a reflexão sobre as atitudes cooperativas e sua prática. As atividades aqui sistematizadas foram realizadas com crianças de 6 a 13 anos, que frequentam o projeto "Escola da Família", inserido em uma escola estadual do município de Americana. O objetivo principal deste trabalho foi contribuir com uma formação educacional que trouxesse o equilíbrio entre competitividade e cooperação, constituindo assim uma prática que vai além da educação escolar. A metodologia utilizada baseou- se nos escritos de Oscar Jara (2006), que em sua tese defende cinco momentos de sistematização para a conclusão de um bom trabalho de campo. São eles: 1) o ponto de partida, que requer a participação ativa dos autores, além de registros de experiências; 2) as questões iniciais que no presente trabalho foram: Qual a compreensão das crianças sobre jogos cooperativos? Essa prática possibilita novos horizontes? Quais são eles? É possível haver um equilíbrio entre cooperação e competição para crianças? Como a prática da cooperação pode ajudar as crianças no desenvolvimento de sua vida social? 3) o resgate do tempo vivido, que busca descrever todo o processo pelo qual passam as atividades e vivências 4) as reflexões de fundo, nas quais se busca aprofundar a compreensão e discussão dos autores e participantes sobre as ações vivenciadas, quais foram todos os processos desenvolvidos. Uma das percepções que a realização desse jogo trouxe é que a cooperação está em nós, só precisamos trabalhar para torná-la evidente; 5) os pontos de chegada, que são a análise de todo o aprendizado no processo. Os resultados finais desta intervenção educativa demonstraram que as crianças apresentaram transformações pequenas, porém significativas no que diz respeito aos seus comportamentos, e às suas práticas com os demais colegas participantes dos jogos e também com as pessoas em geral. Respondendo às inquietações iniciais deste projeto, consideramos que a realização destas atividades trouxe proximidade entre os participantes e ações colaborativas, levando-nos à convicção de que a mudança de comportamento das crianças ultrapassou os limites dos muros da escola.

Palavras-chaves: Jogos Cooperativos, Sistematização da experiência, Educação Popular. 


\section{INTRODUCCIÓN}

Para trabajar el Juego Cooperativo como posibilidad de Educación Popular, este Proyecto de Acción Educativa (PAE) buscó desarrollar actividades dentro de una perspectiva lúdica capaz de ayudar a los niños a ejercitar la reflexión sobre las actitudes cooperativas y su práctica. Las actividades aquí sistematizadas se realizaron los sábados con un grupo variable de alrededor de 25 niños por reunión, totalizando 50 infantes, de 6 a 13 años, durante los meses de aplicación, residentes de la ciudad de Americana, en el barrio São Manoel, entorno donde se ubica la escuela que se encarga del programa 'Escuela de la Familia', la cual se realiza los fines de semana. Estos niños, considerados por la sociedad como un colectivo en situación de vulnerabilidad social, acuden al proyecto para disfrutar de un local de placer donde pueden realizar recreaciones fuera del horario lectivo habitual.

En cuanto a la vulnerabilidad social, Vignoli (2001, citado en Cançado, Cardoso y Souza, 2014 p. 2) la entiende como la falta de acceso a las estructuras de oportunidad que ofrece el mercado, el estado o la sociedad, señalando la falta de un conjunto de atributos necesarios para el uso efectivo de la estructura de oportunidades existente.

La mencionada escuela estatal ejecuta los sábados el programa "Escuela de la familia", que es una política educativa iniciada en 2004, durante el gobierno de Geraldo Alckmin en el estado de São Paulo.

El programa tiene como objetivo reducir la vulnerabilidad de los niños y jóvenes mediante el uso del espacio escolar. Para ello, prevé la apertura de la escuela los fines de semana para la participación de diferentes segmentos sociales en actividades culturales y deportivas, enfocadas en la temática de salud y trabajo (Maia y Ramos, 2018).

La escuela, debido a que no cuenta con recursos ni siquiera con orientación en estas actividades para ser trabajadas con familiares y niños, busca voluntarios que quieran implementar proyectos significativos capaces de contribuir a la formación educativa de los niños y atraer la participación de los familiares, quienes por alguna razón no asisten al programa junto con sus hijos.

Este proyecto de acción educativa nació debido a la necesidad de trabajar con estos niños, con actividades que ayuden no solo a su formación educativa, sino también a su formación social. Así, los juegos cooperativos se plantearon como una posibilidad de la Educación Popular y como un instrumento pedagógico que podría contribuir socialmente a una práctica cooperativa y no solo competitiva. Cabe destacar que durante las actividades, los niños participantes en el proyecto no recibieron premios por jugar ni ningún tipo de recompensa por las actividades; en este proyecto los involucrados tuvieron plena participación única y exclusivamente por el placer de jugar y cooperar juntos.

En esta perspectiva, la práctica de los juegos se pensó como un puente hacia una educación liberadora, que atiende las necesidades de los participantes y valora la vida cotidiana y su cultura.

Brandão (1981) afirma:

Nadie escapa a la educación. En casa, en la calle, en la iglesia o en la escuela, de una o muchas formas, todos involucramos con ella pedazos de la vida: aprender, enseñar, aprender y enseñar. Para conocer, hacer, estar o vivir juntos, todos los días mezclamos la vida con la educación (p. 7). 
De esta forma, el trabajo con juegos cooperativos demuestra que no existe una única forma o modelo de educación; la educación se relaciona con diferentes contextos, formas y temas, incluida la vida cotidiana. Brandão (1981) dice que "la escuela no es el único lugar donde se imparte la educación y puede que ni siquiera sea el mejor sitio; la educación escolar no es su única práctica y el maestro profesional no es su único practicante" (p. 9). Así, este trabajo buscó una práctica pedagógica que transmitiera a través del juego, un ambiente propicio para el aprendizaje que pudiera demostrar a los niños la importancia de la solidaridad, el aprecio, el respeto entre todas las personas y la convivencia.

En esta perspectiva, los juegos cooperativos pueden entenderse como un proyecto basado en el respeto al conocimiento, pero sobre todo como una búsqueda de problematizar una nueva visión en relación a las actividades que emplean los juegos y sus beneficios. Para Oliveira (1985, citado en Sousa y Fávero, 2010), "el juego no solo se inserta en el contexto del deporte en general, sino también en las relaciones sociales, en los conflictos ideológicos, políticos, religiosos, porque a través del juego el individuo, en última instancia, se juzga como 'ser' y no como 'objeto"' (p. 3).

Buscando relacionar juego y cultura, Huizinga (1980), en su obra titulada Homo Ludens, mostró, desde un punto de vista filosófico -mucho más que psicológico o antropológicolos elementos lúdicos presentes en las principales actividades de una sociedad, insertada en la cultura. Sus ideas junto con las de Caillois (1950) nos hacen pensar que el juego está fuertemente ligado al conocimiento.

De esta manera, trabajar con el juego como modalidad de enseñanza, resulta ser un campo de amplio bagaje para el desarrollo histórico-social y para la construcción de una nueva cosmovisión.

Las acciones cooperativas o acciones que valoran mucho la competencia suelen estar vinculadas al entorno social, costumbres, creencias y valores atribuidos por la sociedad. La competencia, según Soler (2008), ganó énfasis en la sociedad moderna cuando la riqueza llegó a ser controlada por solo unos pocos y ellos tenían poder sobre otros. Por eso, hemos competido en lugares, con personas, en momentos que no deberíamos, como si esa fuera la única opción (Brotto, 1999).

De acuerdo a Orlick (1989), experimentos realizados en pequeños grupos muestran que cuando hay un estímulo exacerbado para la competencia, los ambientes se vuelven agresivos y hostiles, ya que la sobrevaloración de lo mejor es perjudicial para el colectivo.

De esta inquietud surge la necesidad de trabajar la cooperación, más que eso, trabajar en juegos cooperativos. En muchas de sus investigaciones, la antropóloga Margarete Mead afirma que en algunas comunidades la competencia aun es una acción desconocida, lo que naturalmente refleja la idea de que tanto la competencia como la cooperación son acciones que dependen de la relación del ser humano con el medio en el que vive y las formas en que se estimulan. Es necesario repensar cómo se dirige al ser humano a competir sin tener realmente una necesidad biológica; como resultado de estas acciones, las sociedades son cada vez más selectivas y desiguales.

Se entiende que los seres humanos somos, por tanto, más cooperativos que competitivos, según defiende Maturana (2004), en su teoría de la cooperación, quien, en oposición a la teoría de la evolución, afirma que solo evolucionamos como especie debido a la cooperación. Menciona, además, que el surgimiento del lenguaje únicamente se expandió a través de la cooperación y que nunca se perpetuaría en el tiempo y en el espacio, sino hubiera cooperación entre los seres. 
Como propuesta de una educación basada en la formación de individuos socialmente cooperativos, este proyecto de acción educativa buscó reflexionar con los estudiantes sobre su rol en la sociedad, y cómo pueden las personas involucradas contribuir con ella; y que con quienes se relacionan no tengan un comportamiento competitivo todo el tiempo, ya que, si bien la competencia es necesaria, no debe ser una conducta esencial o única.

Brown (1995) considera como características de los juegos cooperativos, la confianza, la comunicación y la participación de todos, lo que resulta en la no exclusión. De estas características los juegos cooperativos tienen: la liberación de la competitividad, ya que el interés por el juego viene dado por la participación, no por el deseo de ganar y perder como predica la competencia;

- Libertad de eliminación, ya que el juego busca integrarse e interactuar con todos sin buscar eliminar a ese jugador menos ágil, más débil o menos hábil;

- El lanzamiento para la creatividad, ya que el juego se puede transformar para flexibilizar sus reglas y, junto con la colaboración, permite a los jugadores nuevas creaciones sobre lo que ya existe;

- La liberación de la agresión física, ya que los juegos cooperativos buscan evitar conductas de agresión y falta de respeto que muchas veces son aceptadas y normalizadas en otro tipo de juegos.

Se comienza, entonces, de la idea de que los juegos cooperativos descritos y aplicados son una práctica capaz de transformar socialmente a todo un grupo involucrado y que a partir de este proyecto, los niños son capaces de reflexionar sobre cómo pueden cooperar en todos los ámbitos de la vida y también comprender la razón por la que esta cooperación tiene sentido para ellos.

\section{OBJETIVOS}

Los objetivos de este trabajo fueron:

- Aplicar juegos cooperativos para un grupo de niños y jóvenes, que participan en la Escuela de Familia;

- Analizar los resultados de esta propuesta;

- Sistematizar las aplicaciones de estos juegos cooperativos como puente para el desarrollo de las interacciones sociales, la autonomía, la autoestima y la reflexión sobre el posicionamiento del ser y la pertenencia a una sociedad.

Las actividades pretendían proponer cambios no solo en el contexto escolar, sino en todos los ámbitos de la vida, mostrando que los juegos se pueden trabajar desde una perspectiva no solo competitiva. 


\section{METODOLOGÍA}

Los procedimientos metodológicos partieron de un análisis relacionado con los juegos y el comportamiento de los niños en cuanto a sus prácticas diarias. Así, se pudo observar que la competencia trasciende las múltiples áreas de convivencia social, ya que los niños que participaron en el proyecto demostraron comportamientos altamente competitivos entre sí.

La metodología de investigación se basó en el proceso de sistematización de experiencias de Oscar Jara (2006). La experiencia sistematizada fue el proyecto "Juegos cooperativos: la importancia de jugar 'con' y no solo 'en contra"”, la cual analizó las reacciones de comportamiento y la visión de cooperación de los niños. Según Jara (2006) la sistematización de la experiencia es:

[...] Esa interpretación crítica de una o más experiencias que, a partir de su ordenamiento y reconstrucción, descubre o explicita la lógica del proceso vivido, los factores que intervinieron en dicho proceso, cómo se relacionaron entre sí y por qué lo hicieron de ese modo (p. 24).

Para posibilitar la interpretación crítica, es necesario conocer todos los procesos de la experiencia, en función de la distancia entre ellos. Una vez que la sistematización conduce a una reflexión que hace que la experiencia del proceso vivido se convierta en objeto de estudio e interpretación teórica.

Jara (2006, p. 73) propone que la sistematización de experiencias, como acción metodológica, se realice en cinco etapas, a saber:

1. El punto de partida:

a. Haber participado en el experimento.

b. Tener el registro de experiencias.

2. Las preguntas iniciales:

a. ¿Qué queremos? (Definir el objetivo)

b. ¿Qué experiencias queremos sistematizar? (Delimitar el objeto a sistematizar)

c. ¿Qué aspectos centrales de esta experiencia nos interesa sistematizar? (Definir un eje de sistematización).

3. Recuperación del proceso experimentado:

a. Reconstruir la historia.

b. Ordenar y clasificar la información.

4. Reflexión de fondo:

a. ¿Por qué sucedió lo que sucedió?

b. Analizar, sintetizar e interpretar críticamente el proceso.

5. Puntos de llegada:

a. Formular conclusiones.

b. Comunicar el aprendizaje. 


\section{1. $1^{a}$ ETAPA. PUNTO DE PARTIDA: DIÁLOGO CON NIÑOS Y EXPERIENCIAS PARA EXPERIMENTAR}

La elaboración de este proyecto contó con la participación de los dos autores durante todo el proceso, la experiencia se vivió en todas las fases desde la elaboración, aplicación y evolución.

Cabe señalar que, para tener un trabajo cooperativo verdaderamente válido, la participación de los autores y de los involucrados debe ser integral.

La investigación se basó en el análisis de los materiales: registros escritos y fotográficos, intercambios de experiencias, recuerdos de juegos ya vividos, reflexión sobre los resultados o reglas de los juegos, todo ello elaborado en cooperación con las personas que participaron en el proyecto.

\section{2. $2^{\mathrm{da}}$ ETAPA. PREGUNTAS INICIALES}

Las preguntas iniciales para la investigación de este proyecto fueron: ¿Cuál es la comprensión de los niños sobre los juegos cooperativos? ¿Permite esta práctica nuevos horizontes? ¿Cuáles? ¿Es posible tener un equilibrio entre la cooperación y la competencia para los niños? ¿Cómo puede la práctica de la cooperación ayudar a los niños en el desarrollo de su vida social?

\section{3. $3^{\text {ra }}$ ETAPA. RECUPERACIÓN DE LA VIDA}

Las actividades desarrolladas a lo largo de tres meses se caracterizaron por juegos cooperativos, que se trabajaron a través de prácticas constructivistas.

Aproximadamente 50 niños, de 6 a 13 años, participaron en el proyecto, al igual que los miembros del proyecto "Escuela de Familia", en una escuela estatal en Americana/SP.

El objetivo principal de este proyecto fue construir un puente para el desarrollo de las interacciones sociales, la autonomía, la autoestima y la reflexión sobre el posicionamiento del ser y la pertenencia a una sociedad.

Dentro de esta propuesta abordamos el tema de la cooperación con diferentes juegos, eligiendo a continuación aquellos que tuvieron una relevancia significativa para los cambios de comportamiento:

“Rueda de conversación”: En un primer momento se hizo una breve presentación sobre los participantes de los juegos. Poco después, se plantearon preguntas sobre la comprensión de los niños sobre el tema de los juegos cooperativos, secuenciadas por una presentación de los objetivos de la aplicación de estos juegos.

"Cuerda de la Paz": consiste en un juego en el que se divide una cuerda en dos partes y en medio de ella se coloca una bolsa de dulces y golosinas; luego se dejan dos partes de cuerda, una para cada lado. Se dibuja un círculo en el suelo y se les pide a los niños que formen dos equipos que jugarán juntos, ya que la idea del juego es similar a la 'Cuerda de guerra', pero totalmente invertido en cuanto a su resultado en el que tenemos un equipo ganador y un equipo perdedor. En la 'cuerda de la paz', los niños deben trabajar juntos para hacer explotar la bolsa de dulces, que deberán caer dentro del círculo. En este caso se repartirán entre ellos solo los caramelos que caigan dentro del marcado, el resto que caiga fuera será recogido por los participantes del juego. En el primer intento del juego, 
los niños, acostumbrados a la competitividad, armaron un equipo compuesto por niños mayores por un lado y más pequeños por el otro, incluso con la explicación del juego. En cuanto arrancó, el equipo de niños mayores tiró totalmente de la cuerda provocando que la bolsa explotara y todos los caramelos cayeron fuera del círculo, por lo cual no recibieron caramelos. Se interrumpió el juego y se les preguntó qué había pasado para que no recibieran la recompensa. El objetivo de la pregunta era para que ellos vieran que debían jugar juntos y hacer estallar la bolsa solo cuando estuviera en el centro del círculo, y que sería inútil tirar más por un lado que por el otro. El segundo intento surtió efecto, los niños jugaron cooperativamente y recibieron todos los dulces que compartieron.

Figura 1. Juego: "Cuerda de la Paz".

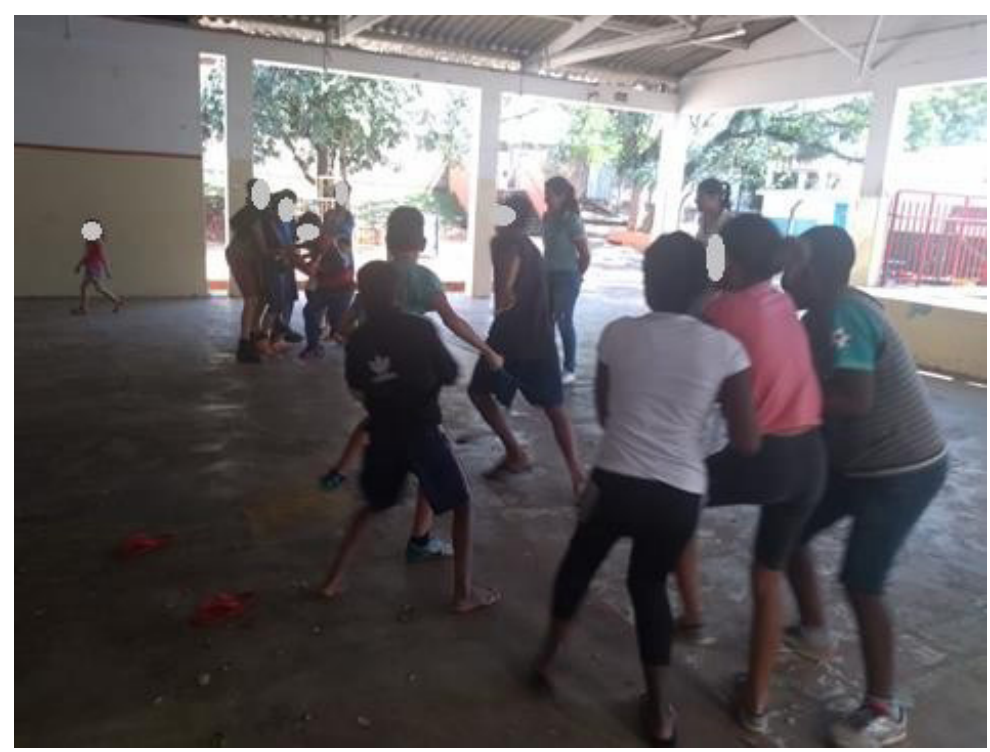

Fuente: Elaboración propia.

"Simón dijo": también se considera un juego cooperativo. Es básicamente un juego de "haz lo que digo, pero no hagas lo que hago". El desarrollo del juego se reduce a hacer que los niños sigan las instrucciones del participante solo cuando dice "Simón dijo", seguido de una orden. Si el participante no dice el nombre "Simón" y solo da la orden, los niños no deben seguir la orden, sino permanecer en la posición en la que se encuentran. Quienes sigan lo que se les preguntó sin que el participante diga: "Simón dijo", en lugar de ser eliminados como en la versión original del juego, en la versión cooperativa, se les invita a ayudar a los otros compañeros que todavía están en el juego. En el primer intento de aplicación, casi la mitad de los niños fueron convidados a ayudar al participante. En el segundo intento, todos estaban muy atentos y emocionados, no querían "perder" para nada. Los que "cometieron errores" y tuvieron que pasar al lado de "ayuda" esperaban que el colega que seguía jugando, cometiera un error. Entonces, cada vez que uno de los jugadores 
fallaba, aplaudían y gritaban sobre el aumento del equipo de ayuda. Se hicieron más de cuatro intentos, ya que les gustó tanto el estilo del juego que siempre que intentábamos pasar al siguiente, decían: "Hagamos esto de nuevo".

Figura 2. Juego: "Simón Dijo".

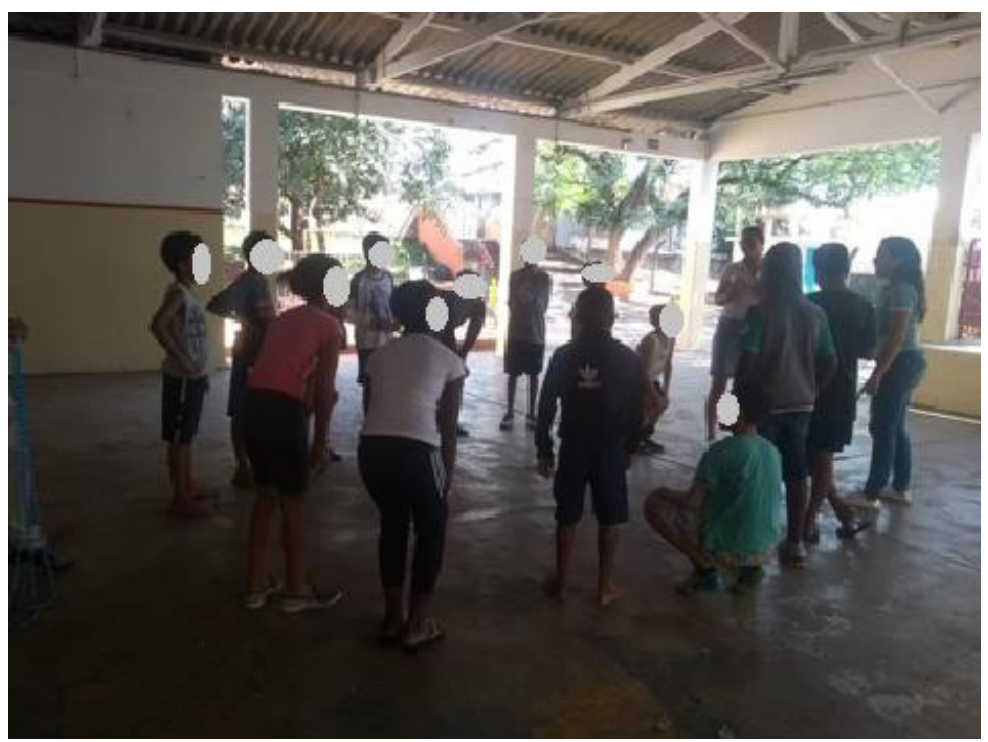

Fuente: archivo personal.

"Conectados": un juego que tiene como objetivo trabajar la interacción con los demás, enfatizando que en el mundo, todos estamos conectados. En este juego, los niños se separan en parejas o tríos y, de espaldas, deben entrelazar los brazos, dando la impresión de ser un solo cuerpo. La idea es que todos los grupos caminen una cierta distancia, superando juntos los obstáculos que se colocan en el camino. Para este juego, es necesario utilizar conos, cuerdas y palos para colocar barreras y tizas para trazar el camino que deben seguir los niños. El pequeño equipo nunca podrá separarse, por más difícil que sea pasar las etapas del juego, lo que a su vez no permite que los dos participantes pongan los pies juntos en el suelo; por lo tanto, mientras uno pasa por el obstáculo, el otro debe ser llevado en la espalda, pero para que ambos puedan cruzar la barrera, el compañero que estaba en la espalda se queda en el piso y cruza al amigo que lo cargó. Inicialmente, las parejas, acostumbradas a los juegos siempre competitivos, trazaban el recorrido en marcha, con la expectativa de ganar el boleto para convertirse en 'ganadores'. Así que se propuso que la pareja que lograra cruzar los obstáculos primero, regresara y asistiera a la que todavía estaba en la ruta. Como resultado, fueron muy cooperativos. Cada vez que una pareja cruzaba la línea de meta, pronto regresaban para ayudar a los demás a cruzar también. Para que todos pudieran participar en el juego, los niños mayores crearon una forma de cruzarse con los más pequeños. Durante la aplicación de este juego, se notó la emoción y concentración 
de los niños al ayudar al amigo a cruzar las barreras y completar el recorrido sin soltarse. Una de las percepciones que trajo la realización de este juego es que la cooperación está en nosotros, solo tenemos que trabajar en ello para que sea evidente.

"Pasar el balón con los pies": este juego tiene como objetivo trabajar la coordinación y la destreza de los pies en conjunto, para que todos puedan tener la percepción de que para lograr unos objetivos es necesaria la participación del otro. Por tanto, la idea del juego es que los niños formen una cadena de cuerpos tendidos en el suelo uno al lado del otro, con los pies en alto, para que puedan acomodar la pelota cuando la atraviese. Luego, el primer participante recibe el balón con los pies y lo pasa al siguiente, sin dejarlo caer, hasta que llega al último. Si dejan caer la pelota durante el recorrido, deben regresar al comienzo de la línea y reiniciar el cruce; cuando el último participante recibe la pelota, debe levantarse y correr hasta el inicio de la línea y comenzar de nuevo todo el proceso. Durante la aplicación de este juego en particular, los niños se mostraron muy agitados y sin interés en cooperar con el desarrollo de la actividad, por lo que el juego no salió como se esperaba. Fue necesario interrumpirlo y plantear preguntas sobre la posición de los niños y la forma en que se desarrollaba el juego. Después de todos los ajustes, los participantes no solo se cuestionaron sobre el objetivo de la actividad, sino también, cuál era la idea principal de transformación que podía dejar a los niños; entonces, se re hizo el juego, pero con las propuestas planteadas por parte de los participantes, quienes dijeron que el juego sería mucho más divertido, si pudieran seguir pasando el balón, aunque se cayera, sin tener que empezar de nuevo, ya que este siempre cae y casi nunca llega al último participante. Se hizo la modificación sugerida y todos empezaron a trabajar en equipo.

"Dinámica de bastones": este juego pretende contribuir a la visión de los niños de ayudar y posicionarse en el lugar de los demás. El juego consiste en la idea de que cada niño debe tener un bastón en la mano y colocarse uno al lado del otro hasta formar un círculo. Cuando ya están todos en su lugar, los bastones deben permanecer estacionarios mientras los jugadores corren en círculo, sosteniendo el siguiente bastón, sin dejar que caiga al suelo. Si un bastón cae, los jugadores deben retirar uno de los bastones del juego. El desarrollo de esta actividad trajo una gran participación por parte de los niños, quienes mostraron mucho interés por el juego. Se pudo identificar que los participantes se esforzaron por no dejar caer los bastones y prestaron total atención durante todo el proceso. Esta actividad en sí trajo la reflexión de que los niños siempre están con los brazos abiertos para aprender nuevas prácticas y tienen sed de conocimiento. Al final del juego, pudieron exponer sus opiniones e informaron que el hecho de tener que sostener el bastón del amigo para evitar que se le cayera era emocionante, porque de esa manera necesitaban estar conectados, algo que no ocurre muy a menudo en los juegos competitivos habituales.

"Hula-hop en el cono": Este juego cooperativo busca trabajar con los niños la coordinación motora, la paciencia, la concentración y la determinación. Es un juego muy divertido en el que se forman dos equipos de niños; cada uno de ellos tiene un cono como línea de salida, otro cono a aproximadamente 10 metros como "blanco", y un hula hoop para lanzar. Los equipos formados deben hacer fila, y el juego debe comenzar con el primer participante de cada equipo. La idea es hacer que el hula- hop pueda "enlazar" el cono sin dejarlo caer. Cada niño debe tener un solo movimiento. Cuando lance, el hula-hop debe entrar en el cono, pero si el tiro se pierde, el jugador deberá correr para atraparlo y entregárselo al siguiente jugador en la fila. Luego, los niños deben ir tirando los aros de hula-hop hasta que suelte el último. En el juego, si el cono cae al intentar hacer un bucle, el jugador que 
lanzó el hula-hop debe levantarlo. Este juego fue muy apreciado por los niños y contó con la colaboración en todo momento, ya que los jugadores estaban juntos para ayudarse en el lanzamiento; además, cuando algo se salía del plan, se observó que siempre estaban dispuestos a ayudar a ese amigo que perdió el tiro o dejó caer el cono.

Figura 3. Juego: "Hula-hop en el cono".

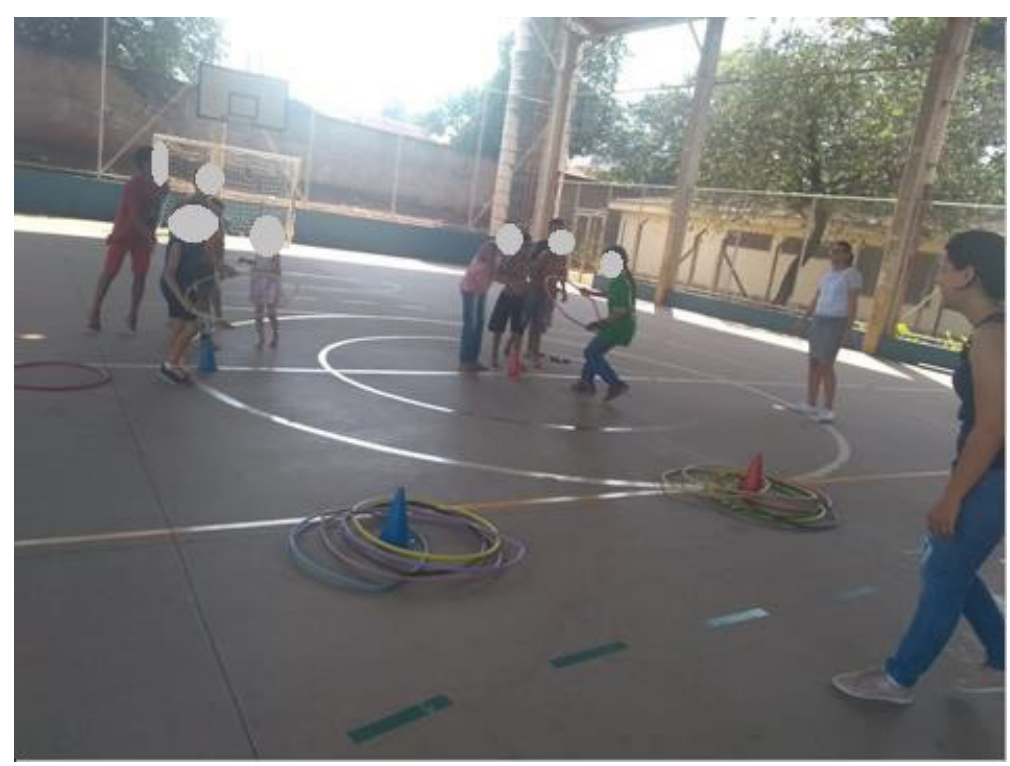

Fuente: archivo personal.

“Rueda de cierre": Al final de cada día de encuentro con los niños, se formó un círculo de conversación para reflexionar sobre los juegos cooperativos y cómo se sintieron durante las actividades. Las respuestas fueron muy diversas. Algunos niños dijeron que siempre preferían tener un ganador y un perdedor; otros, sin embargo, informaron sobre la importancia de cooperar y tener la oportunidad de ganar o perder siempre, pero que sería un resultado para todos, para que nadie estuviera triste o feliz solo.

Soler (2008) también reafirma que la práctica de juegos cooperativos desarrolla la promoción de la autoestima y la convivencia con el fin de prevenir problemas sociales. Por tanto, los juegos cooperativos, para Costa y Pimentel (2007, p. 4), deben ser trabajados de manera "[...] que sensibilice a los estudiantes sobre las injusticias sociales vigentes en la sociedad, sin embargo, es un contexto que se puede transformar". Así, la realización de este proyecto de acción educativa permitió comprender que el trabajo con juegos cooperativos trae pequeños cambios inicialmente, pero constantes, ya que se desarrollan a diario y con total dedicación. 


\section{4. $4^{\text {ta }}$. ETAPA. REFLEXIÓN DE FONDO}

Los Juegos cooperativos realizados a lo largo de todos los encuentros, nos lleva a reflexionar sobre todas las acciones desarrolladas que posibilitaron una nueva mirada sobre la práctica de la cooperación, haciéndonos resumir las preguntas que inicialmente motivaron este trabajo y una discusión sobre los cambios de comportamiento y valores de los involucrados en el proyecto.

Según Orlick (1989), a los niños no se les enseña a disfrutar de la búsqueda de conocimientos: se les enseña a esforzarse por obtener altas calificaciones. Asimismo, no se les enseña a que les guste el deporte por el placer del juego, se les enseña a disfrutar ganando, de forma primordialmente competitiva, es decir, unos contra otros.

Los círculos de conversación, como dicen Silva y Vasconcelos (2019), son capaces de traer ricas experiencias y reflexiones, pues a partir de ellas se puede vislumbrar una realidad que muchas veces no se asume en las escuelas. Por tanto, los juegos, en este contexto, sirvieron para problematizar la práctica de la cooperación, como herramienta educativa capaz de modificar acciones y comportamientos. Llevar a todos a reflexionar sobre las experiencias vividas durante los encuentros y los resultados obtenidos.

En el juego "La cuerda de la paz", los niños, aun con comportamientos enteramente competitivos, buscaron caminos que los beneficiaran solo a ellos mismos, sin tomar en cuenta a otros amigos, por lo que tuvieron el resultado de una acción negativa, que los llevó a reflexionar sobre el respeto por las reglas y el comportamiento que debían tener durante el juego para que el resultado pudiera beneficiar a todos. Para que las acciones sean cooperativas, según Teixeira (2005) es necesario que los individuos tengan alineados sus sentimientos, pensamientos y acciones, en la búsqueda de una relación sana para una sociedad mejor.

Luego de analizar sus comportamientos, los niños pudieron ver que actuar en cooperación es mucho más fácil para lograr sus objetivos, por lo que reflexionaron que nadie necesitaba sobrepasar los límites del otro para lograr un buen resultado, y estas acciones son reflejos que podrían llevar más allá de los juegos: en el/la escuela, con sus familias y la sociedad en general.

Orlick (1989) señala:

Uno de los mitos que ha sido derribado por la investigación sobre el aprendizaje cooperativo es que la competencia es necesaria para que los estudiantes aprendan o se desempeñen bien. Con respecto al rendimiento académico, se encontró que los niños se desempeñan igual de bien en clases cooperativas como competitivas (p. 27).

En la secuencia, veremos una tabla, indicada por Brotto (1999, p. 56), que relaciona los juegos competitivos con los juegos cooperativos. El cuadro es una parte de la obra de Zimarian Walker, en el libro Educando para la paz (1987). 
Tabla 1. Juegos competitivos y juegos cooperativos

\begin{tabular}{|c|c|}
\hline Juegos competitivos & Juegos cooperativos \\
\hline Son divertidos apenas para algunos & Son divertidos para todos \\
\hline $\begin{array}{l}\text { Algunos jugadores tienen sentimientos de } \\
\text { derrota. }\end{array}$ & $\begin{array}{l}\text { Todos los jugadores tienen sentimientos de } \\
\text { victoria. }\end{array}$ \\
\hline $\begin{array}{l}\text { Algunos jugadores quedan excluidos por su } \\
\text { falta de habilidad. }\end{array}$ & $\begin{array}{l}\text { Todos se involucran independientemente de su } \\
\text { capacidad. }\end{array}$ \\
\hline $\begin{array}{l}\text { Se aprende a ser desconfiado, egoísta sin } \\
\text { sentirse apenado por los demás. }\end{array}$ & Se aprende a compartir y a confiar. \\
\hline $\begin{array}{l}\text { División por categorías: niños x niñas, creando } \\
\text { barreras entre las personas, justificando las } \\
\text { diferencias como una forma de exclusión. }\end{array}$ & $\begin{array}{l}\text { Hay una mezcla de grupos que juegan juntos } \\
\text { creando un alto nivel de aceptación mutua. }\end{array}$ \\
\hline $\begin{array}{l}\text { Los perdedores quedan fuera del juego y } \\
\text { simplemente se convierten en observadores }\end{array}$ & $\begin{array}{l}\text { Los jugadores están involucrados en los juegos } \\
\text { para pedidos mayores, tomando más tiempo } \\
\text { para desarrollar sus capacidades. }\end{array}$ \\
\hline $\begin{array}{l}\text { Los jugadores no se solidarizan y se alegran } \\
\text { cuando algo "malo" ocurre a los otros. }\end{array}$ & $\begin{array}{l}\text { Se aprende a simpatizar con los sentimientos } \\
\text { de los demás, deseando también su éxito. }\end{array}$ \\
\hline Los jugadores son desunidos. & $\begin{array}{l}\text { Los jugadores aprender a tener un sentido de la } \\
\text { unidad. }\end{array}$ \\
\hline $\begin{array}{l}\text { Los jugadores pierden la confianza en sí mismo } \\
\text { cuando son rechazados y cuando pierden. }\end{array}$ & $\begin{array}{l}\text { Desarrollan auto confianza porque son } \\
\text { aceptados. }\end{array}$ \\
\hline $\begin{array}{l}\text { La poca tolerancia a la derrota desarrolla en } \\
\text { algunos jugadores un sentimiento de rendición } \\
\text { ante las dificultades. }\end{array}$ & $\begin{array}{l}\text { Se refuerza la capacidad de perseverar ante las } \\
\text { dificultades. }\end{array}$ \\
\hline Pocos tienen éxito & $\begin{array}{l}\text { Todos encuentran la manera de crecer y } \\
\text { desarrollarse. }\end{array}$ \\
\hline
\end{tabular}

Fuente: Brotto (1999, p. 56).

Dentro de esta perspectiva, se entiende que los niños inicialmente presentaron acciones competitivas en relación con los juegos, pero durante estos fueron absorbiendo actitudes cooperativas, ya que la competencia no terminó, sino que fue equilibrada.

La cooperación puede ser una herramienta importante en el desarrollo de la interacción social de los niños, según Soler (2008, p. 28):

El juego es una forma extremadamente poderosa de formar comportamientos. También podemos, a través de este, modificar una sociedad, haciéndola más humana, cooperativa y pacífica o, por el contrario, haciendo que esa misma sociedad sea extremadamente competitiva, violenta e inhumana. Debemos recordar que el juego puede llevarnos en diferentes direcciones.

Cabe destacar la importancia de trabajar la cooperación en la búsqueda de una sociedad mejor, donde sus individuos sepan cooperar y ser más pacíficos. 
Es menester mencionar que aunque se están presentando de manera antagónica, los juegos cooperativos y competitivos pueden combinarse en prácticas pedagógicas en diferentes categorías.

A continuación, se enumeran algunos ejemplos establecidos por Orlick (1989, citado en Soler, 2008, pp. 26-27):

a) Juegos cooperativos sin perdedores: todos los participantes forman un gran equipo. Son juegos totalmente cooperativos.

b) Juegos de resultado colectivo: permiten la existencia de dos o más equipos. Hay un fuerte rasgo de cooperación dentro de cada equipo y también entre equipos. El objetivo principal es lograr metas comunes.

c) Rotación: los jugadores cambian de bando según situaciones preestablecidas, tales como: después del saque (voleibol); después de un saque de esquina (fútbol, balonmano); tan pronto como lance un tiro libre (baloncesto)

1. Inversión de puntuación: el jugador que anota el punto pasa al otro equipo.

2. Inversión de la puntuación: el punto conseguido lo anota el otro equipo.

3. Inversión total: tanto el jugador que hizo el punto, como el punto logrado, pasa al otro equipo.

d) Juegos semi-cooperativos: adecuado para comenzar a trabajar con juegos cooperativos, especialmente con adolescentes, en un contexto de aprendizaje deportivo. Ofrece la oportunidad a los participantes de jugar en diferentes posiciones:

1. Todos juegan: todos los que quieran jugar, tienen el mismo tiempo de juego.

2. Todos tocan/todos pasan: el balón debe pasarse entre todos los jugadores del equipo para que se valide el punto.

3. Todos los puntos anotados: para que un equipo gane, todos los jugadores deben haber anotado al menos 01 puntos durante el juego.

4. Todas las posiciones: todos pasan por las diferentes posiciones del juego (portero, entrenador, aficionado, técnico, etc.)

5. Pase mixto: el balón debe pasarse alternativamente entre chicos y chicas.

6. Resultado mixto: los puntos son convertidos, a veces por una niña, a veces por un niño.

Añadiendo a las categorías de los Juegos Cooperativos, Brotto (1999), menciona una visión de juego:

Por supuesto, no existe una receta milagrosa para la felicidad. Sin embargo, existen claves para orientar el camino hacia una mejor convivencia y bienestar común. El paso a paso en este camino es siempre un paso, es decir, se construye a través del juego de las relaciones interpersonales y grupales, dada la necesidad de resolver problemas, armonizar conflictos y lograr metas (p. 60). 


\section{5. $5^{\text {ta }}$. ETAPA. PUNTOS DE LLEGADAS}

Los puntos de llegada de esta investigación al final del curso demuestran que los niños mostraron cambios pequeños, pero significativos en cuanto a sus comportamientos y prácticas con otros compañeros que participaban en los juegos, y que la realización de este proyecto trajo cercanía entre los niños participantes y los investigadores, además de acciones colaborativas durante el desarrollo de actividades.

Para nosotros como futuros educadores es cierto que los niños, como todos, somos seres en construcción, llenos de conocimientos e historias.

Por tanto, corresponde, a nosotros educadores, promover actividades que estimulen a los niños a sentirse impulsados a tener dominio sobre todas y cada una de las áreas del conocimiento, y que las prácticas de los contenidos sean capaces de cambiar el pensamiento o la actitud del niño.

Las actividades brindaron experiencias y acciones que establecieron y abrieron posibilidades de asociación, respeto, cariño y amor.

Respecto a la palabra Amor, Maturana y Verden-Zoller (2004), entienden:

El amor es emoción, la disposición corporal dinámica que constituye en nosotros la operatividad de acciones de convivencia en mutua aceptación en cualquier dominio particular de relaciones con otros seres, humanos o no. La biología del amor es fundamental para el desarrollo de cada individuo (p. 134).

Para Brandão, cuando aprendemos a amar, queremos dar amor al otro, compartir esta pura y rica manifestación de sentimientos. Este proyecto permitió conocer la práctica de la cooperación y sus beneficios a través de las actividades aplicadas, demostrando que los niños tienen sed de conocimiento y son capaces de aprender y practicar experiencias compartidas.

Incluso con la finalización de la investigación debido a la pandemia mundial de Covid-19, estamos seguros de que logramos plantar una pequeña semilla en los niños. Brandão (1981) señala:

La educación existe donde no hay escuela y en todas partes pueden existir redes y estructuras sociales para transferir conocimientos de una generación a otra, donde todavía no se ha creado la sombra de algún modelo de enseñanza formal y centralizado, porque la educación aprende del hombre para continuar el trabajo de la vida (p. 12).

De esta forma, los juegos cooperativos fueron una propuesta educativa que permitió un intercambio de experiencias que sin duda va más allá de los muros de la escuela. Desde este estudio reflejamos que la Educación Popular en este contexto siempre será progresiva, mostrando nuevos horizontes de acción y trabajo.

\section{REFERENCIAS BIBLIOGRÁFICAS}

Brandão, C. R. (1981). O que é educação. São Paulo: Brasiliense.

Brotto, F. O. (1999). Jogos cooperativos: o jogo e o esporte como um exercício de convivência. Campinas, SP: [s.n.]. 
Brown, G. (1995). Jogos cooperativos: teoria e prática. 2. ed. São Leopoldo: Sinodal.

Freire, P. (1996). Pedagogia da Autonomia: saberes necessários à pratica educativa. São Paulo: Paz e Terra.

Cançado, Taynara Candida Lopes, Cardoso, Cauan Braga da Silva \& Souza, Rayssa Silva de. (2014). Trabalhando o conceito de Vulnerabilidade Social. Disponível em: http://www.abep.org. br/ abeporgb/abep.info/files/trabalhos/trabalho_completo/TC-10-45-499- 410.pdf> Acesso em: 28 out. 2020.

Costa, Ivanete Maria da; Pimentel, Giuliano Gomes de Assis (2007). Reflexões críticas sobre o uso de jogos cooperativos em escolas com violência discente. Disponível em: <http://www. gestaoescolar.diaadia.pr.gov.br/arquivos/File/producoes_pde/artigo_ivanete_maria_costa.pdf> Acesso em: 11.dez.2020.

Jara Holliday, Oscar. (2006). Para sistematizar experiências. Disponível em: <https://docplayer.com. br/24615-Para-sistematizar-experiencias-oscar-jara-holliday.html> Acesso em: 11 dez. 2020.

Maia, Gabriela Guillardi da Silva; Ramos, Géssica Priscila. (2018). Programa Escola da Família: a Escola a Desserviço da Escola. Educação em Revista Marília, 19(1), 103-120, Jan-Jun, 2018.

Disponível em: <http://revistas.marilia.unesp.br/index.php/educacaoemrevista/article/view/7904> Acesso em: 24. Ago. 2019.

Maturana, H. \& Verden-Zoller, G. (2004). Amar e brincar: fundamentos esquecidos do humano. São Paulo: Palas Athena.

Oliveira, V. M. (1985). Educação Física Humanista. Rio de Janeiro: Ao livro técnico. En Sousa, Diego Petyk de; Fávero, Maria Teresa Martins. Educação física na perspectiva dos parámetros curriculares nacionais para o ensino fundamental. Disponível em: < https://www.efdeportes. com/efd147/educacao-fisica-na-perspectiva-dos-parametros-curriculares-nacionais.htm> Acesso em: 11.dez.2020.

Orlick, T. (1989). Vencendo a competição. São Paulo: Círculo do Livro.

Soler, R. (2008). Brincando e aprendendo com os jogos cooperativos. 2. ed. Rio de Janeiro: Sprint.

Silva, Keila Mourana Marques \& Vasconcelos, Valéria Oliveira. (2019). As rodas de conversa como instrumento metodológico na Educação de Jovens e Adultos. EJA em Debate, Santa Catarina, Ano 8, n. 13, Jan/ Jun. 2019.

Teixeira, M. (2005). Ser cooperativo. Revista Jogos Cooperativos, Sorocaba, (2). 